\title{
Tattoos aus dem Regenwald: \\ Die magische Indianertinte aus Genipa americana
}

\author{
Michael Schwerdtfeger
}

\begin{abstract}
The fruit juice of Genipa americana (Rubiaceae) - known as genipapo, huito, or jagua - has long been used by indigenous peoples of South and Central America for staining the skin. The black markings painted on the body resemble ,real "tattoos. The stain is temporary for about two weeks and cannot be removed during this time. The plant and its use are described here along with a discussion of cultural, psychological, and social aspects of tattoos.
\end{abstract}

\section{Zusammenfassung}

Genipa americana, u. a. auch als Jagua bezeichnet, ist ein Rötegewächs, dessen Fruchtsaft Haut temporär schwarz färbt. Dementsprechend lassen sich mit Jagua-Saft kurzlebige, in dieser Zeit aber nicht entfernbare Zeichnungen, die echten gestochenen Tattoos sehr ähnlich sehen, auf die Haut aufbringen. Biologie und Verwendungsmöglichkeiten von Jagua werden vorgestellt. Außerdem wird ein Überblick über kulturelle, psychologische und soziologische Aspekten von Tattoos gegeben.

\section{Das Märchen vom kleinen blassen Jungen}

Vor Zeiten, in der artigen Wirtschaftswunderwelt der Sechziger Jahre, war einmal ein kleiner Junge, der sich für Pflanzen und Tiere mehr interessierte als für alles andere. Seine Freunde waren Bücher, und seine guten Eltern und Großeltern schenkten ihm so viele, wie er nur haben wollte. Und weil er blitzgescheit reden konnte, alle Tiere auswendig kannte und, anders als die anderen Kinder, immer ordentlich in Schlips und Kragen ging, wurde er alsbald der kleine Herr Professor geheißen. Es stand außer Zweifel, dass er einmal an der Universität studieren, Latein reden und ein großer Gelehrter werden würde.

Was hinter dem ordentlichen Seitenscheitel, dem blassen Stubenhockerteint, unter Oberhemd und Pullunder niemand vermutete: Der Junge hatte, er wusste selber nicht wieso, noch eine andere Leidenschaft. Er bewunderte die Hippies mit ihren Blue Jeans, den wilden Haaren, dem sonnenbraunen Teint - und ihren coolen Tattoos (die man damals noch verschämtdistanziert als Tätowierungen bezeichnete). Aber seine Eltern klärten ihn auf, dass das Gammler seien und dass so etwas nur alte Seeleute, Kriminelle oder englische Soldaten hätten, dass man solche Dummheiten nie mehr los wird und auf jeden Fall sein ganzes Leben lang bereut.
Aber es half alles nichts: Mehr und mehr wuchs die heimliche Faszination am Tattoo, und verschämt malte er sich aus, selber einmal so etwas Cooles zu haben. Aber ach: Für ihn als angehenden seriösen Akademiker würde dieses Lebensgefühl Tattoo wohl immer ein unerfüllbarer Traum bleiben.

\section{Begegnungen in Ecuador}

Viele Jahre später: Das Biologiestudium hatte ich mit dem Diplom abgeschlossen und fand mich in einer kleinen Forschungsstation im Tieflandregenwald des ecuadorianischen Amazonasgebietes wieder, wo ich den jungen Forstingenieur Nixon Revelo kennenlernte. Er hatte hier einen Tropengarten angelegt, wo er unter anderem die herrlichsten Helikonien kultivierte. Und er zeigte uns Stationsgästen noch etwas anderes: In einer Schale hatte er Huito, den Saft aus der Frucht des Baumes Genipa americana. Der darin enthaltene Wirkstoff Genipin geht mit den Eiweißen der obersten Hautschicht (Epidermis) eine chemische Reaktion ein, färbt die Haut irreversibel blauschwarz und erzeugt so ein Tattoo, das sich mit nichts abbekommen lässt - bis es im Zuge der Erneuerung der obersten Hautschicht nach ca. $14 \mathrm{Ta}$ gen herausgewachsen ist. Wie cool, dachte ich, temporäre Tattoos für die Dauer einer Regenwaldexkursion; das Lebensgefühl Tattoo zum 


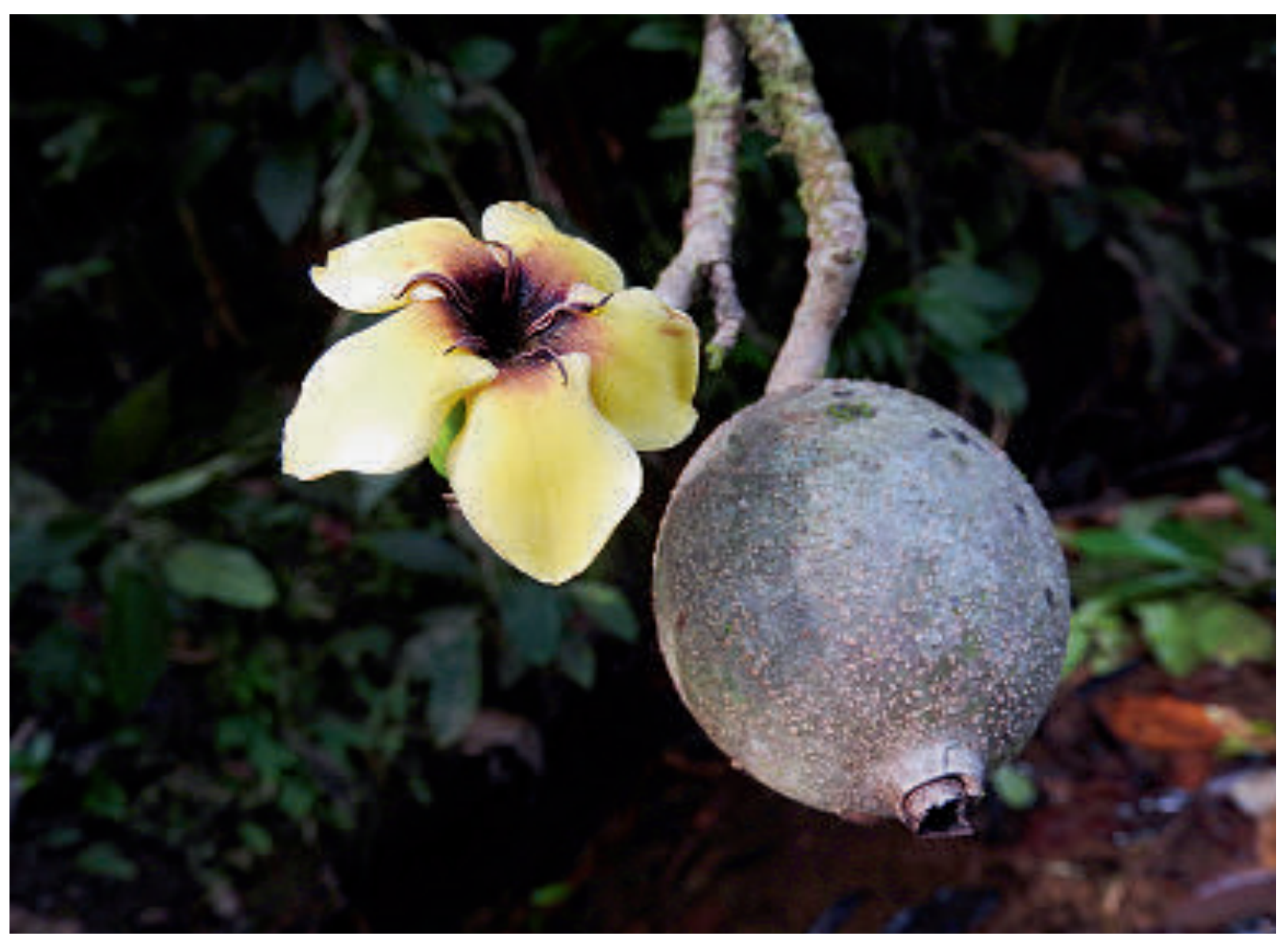

Ausprobieren! Konnte Pachamama (das ist in der Sprache Quichua so etwas wie Gottvater und Mutter Natur in einer Person!) einem jungen Biologen, unter dessen Akademikerschale ein tattoophiles Herz schlägt, ein größeres Geschenk reichen? Ich musste mit ansehen, wie die jungen Leute auf der Station sich unbekümmert überall mit albernen Bildchen bekritzelten und war traurig: ein Tattoo ist doch etwas Bedeutendes, Feierliches, und ich würde mir damit gewiss mehr Mühe geben!

$\mathrm{Ob}$ ich auch ein Tattoo wolle. „Nein, nein, danke!" log ich, denn man mag es kaum glauben: Ich war damals noch so gehemmt und verklemmt, dass ich es nicht einmal auszuprobieren wagte.

Die botanischen Lehr- und Wanderjahre gingen vorüber, und es ist mir in jeder Hinsicht gut ergangen: Promotion, Daueranstellung im Traumberuf, Ehe und Familie, Haus und Gar-

Abb. 1: Blüte und Frucht von Genipa americana. ten, Freundeskreis und Hobbies. Aber ich habe nie vergessen, dass „da draußen“ in den endlosen Wäldern Amazoniens meine Tattoo-Pflanze wächst. Genipa americana wird ein hoher Baum, kommt unter Glas nicht zur Blüte und wird daher in Botanischen Gärten fast nicht kultiviert. Die Pflanze und ihr magischer Saft waren also für mich unerreichbar - so unerreichbar wie ein cooles Tattoo für einen ehrenwerten Akademiker in den besten Jahren.

\section{Wiedersehen in Costa Rica}

Zweiundzwanzig Jahre später feierte ich mit meiner flüchtigen Reisebekanntschaft Genipa ein unerwartetes Wiedersehen. Ich bereitete meine jährliche Studentenexkursion nach Costa Rica vor und stieß bei den Recherchen über sekundäre Pflanzenstoffe darauf, dass eine Firma in den USA den Saft unter dem Namen Jagua verkauft. Das Zeug ist erhältlich! Was wäre wenn ...?

Damals in Ecuador hatte ich mich nicht getraut; vielleicht würde ich mich jetzt trauen. 

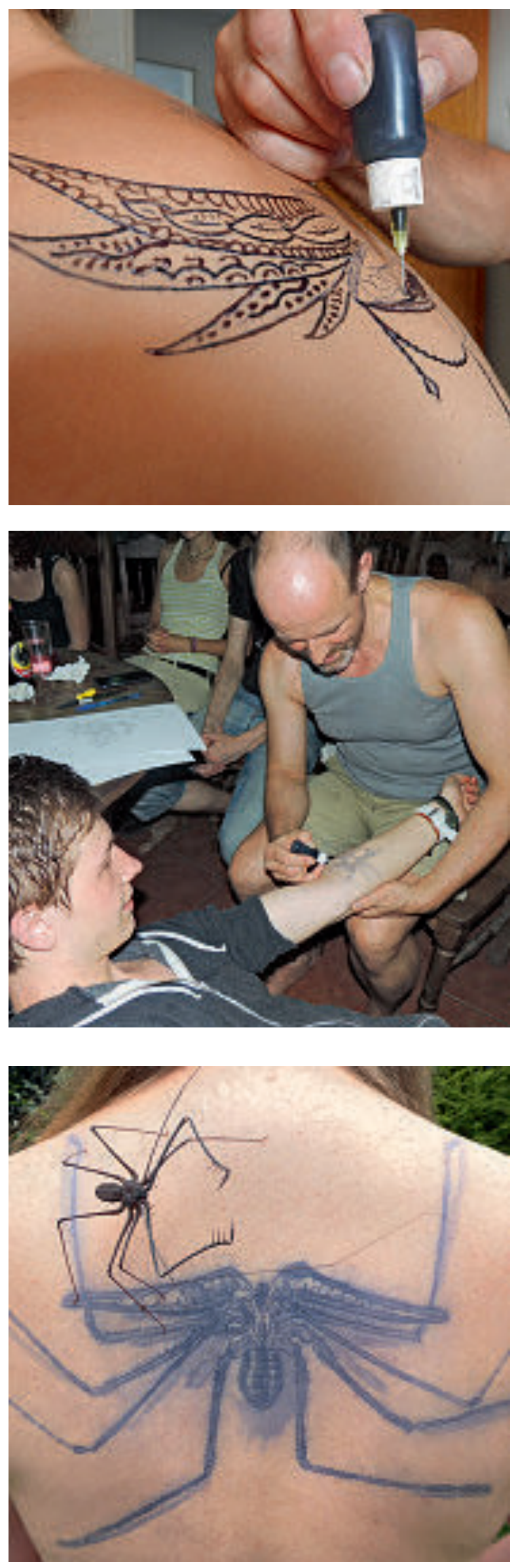

Was würden die Studenten sagen, wenn der kultivierte Universitätsdozent zur Exkursion mit Tattoos auftaucht?

Die Studenten waren schwer begeistert von der lebensgroßen Vogelspinne auf meiner sportlichen Wanderer-Wade. „Eine Erinnerung an Ecuador 1998“, erklärte ich mit der größten Selbstverständlichkeit. Schweißtreibende Regenwaldmärsche, Waten in Flussbetten, Baden in heißen Quellen, in den Mangroven und im Pazifik - alles machte mein Tattoo mit. Natürlich wusste niemand von Genipa, wie hätte man also darauf kommen sollen, dass es kein echtes Tattoo, sondern „nur gemalt“ war?

Nach einigen Tagen hielt ich den Teilnehmern einen kleinen Vortrag über die Chemie des Regenwaldes und das Potenzial der Vielfalt sekundärer Pflanzenstoffe und lüftete das Geheimnis. Wie erwartet, waren die jungen Leute schwer begeistert, und an den folgenden Abenden ließen sich viele von mir so ein RegenwaldTattoo zeichnen. Auch die übrigen Gäste der Station, Wissenschaftler aus Deutschland, Österreich und Luxemburg, aus Kolumbien und den USA wurden neugierig und vereinbarten einen Termin, und ich konnte stolz bemerken, dass ich in meiner neuen Karriere als Tätowierer ,international arbeitete“.

Nach meiner Rückkehr erzählte ich meiner Frau die Story und stellte ihr mein neues Tattoo vor. Und obwohl meine Frau bisher weder besonders auf Tattoos noch auf Spinnen stand, ließ sie sich von dem Witz und dem Charme der Geschichte anstecken. Und weil ich mich mittlerweile mit dem schicken Hingucker immer wohler fühlte, habe ich das Spiel zu Hause weitergespielt, das Tattoo alle 14 Tage, wenn es blass wurde, heimlich wieder nachgezogen und so meinen gesamten Freundes- und Kollegen-

Abb. 2 (oben): Die Farbe wird auf die Haut aufgebracht. Abb. 3 (Mitte): Eine Vogelspinne entsteht auf einem Arm. Abb. 4 (unten): Vorbild und Jagua-Tattoo einer Geißelspinne der Gattung Heterophrynus.

Abb. 5 (Seite 131): Vogelspinne auf der Wade des Autors. 
kreis mit meinem neuen Tattoo verblüfft. Wie habe ich es genossen, das es jeder für echt hielt und mir jede noch so verrückte Geschichte, die ich dazu auftischte, abgekauft wurde.

Nach Ablauf eines ganzen herrlichen KurzeHosen-Sommers habe ich dann aufgehört, sie nachzuziehen: Ich habe sie mir endlich stechen lassen. Und so hat sich für den kleinen blassen Jungen von damals - besser spät als nie - der langgehegte Traum vom Tattoo doch noch erfüllt.

\section{Fakten zu Genipa americana}

Genipa americana L. ist ein Baum aus der Familie der Rötegewächse, dessen natürliche Verbreitung das gesamte tropische Amerika von Mexico über die Karibik bis Brasilien und Bolivien umfasst. Ökologisch ist die Art recht flexibel und kommt in immergrünen Tieflandregenwäldern wie in Trockenwäldern vor. In der Natur wird die Art ein bis zu 15 m hoher Baum mit gutem, hartem Holz. Alle Teile der Pflanze enthalten pharmazeutisch wirksame Substanzen mit bakteriziden und fungiziden Eigenschaften, wirken entzündungshemmend und fiebersenkend und spielen daher in der Volksmedizin vieler indigener Ethnien eine große Rolle. Die duftenden Blüten entstehen an wenigblütigen Rispen, sind honiggelb und tragen im Zentrum ein braunviolettes Saftmal. Die Früchte, die in Form und Größe zwischen $\mathrm{Zi}$ trone und Orange liegen, enthalten um die flachen Samen herum ein saftiges Fruchtfleisch und werden zu Getränken und Eis, Konfitüre und Spirituosen verarbeitet. Die prominenteste Eigenschaft ist jedoch, dass der Saft der Früchte die menschliche Haut blauschwarz färbt und daher seit Urzeiten von den verschiedensten indigenen Völkern von Costa Rica bis Bolivien zum Schmücken des Körpers verwendet wird.

\section{5. „Kriegsbemalung“ - Die Indigenen Amerikas und ihr Körperschmuck}

Als die Kinder zum Fasching noch als „Cowboy und Indianer" gingen, durfte neben Kopfschmuck, Pfeil und Bogen auch die „Kriegsbemalung" nicht fehlen. Für uns zivilisierte Europäer, deren Etikette seit Jahrhunderten

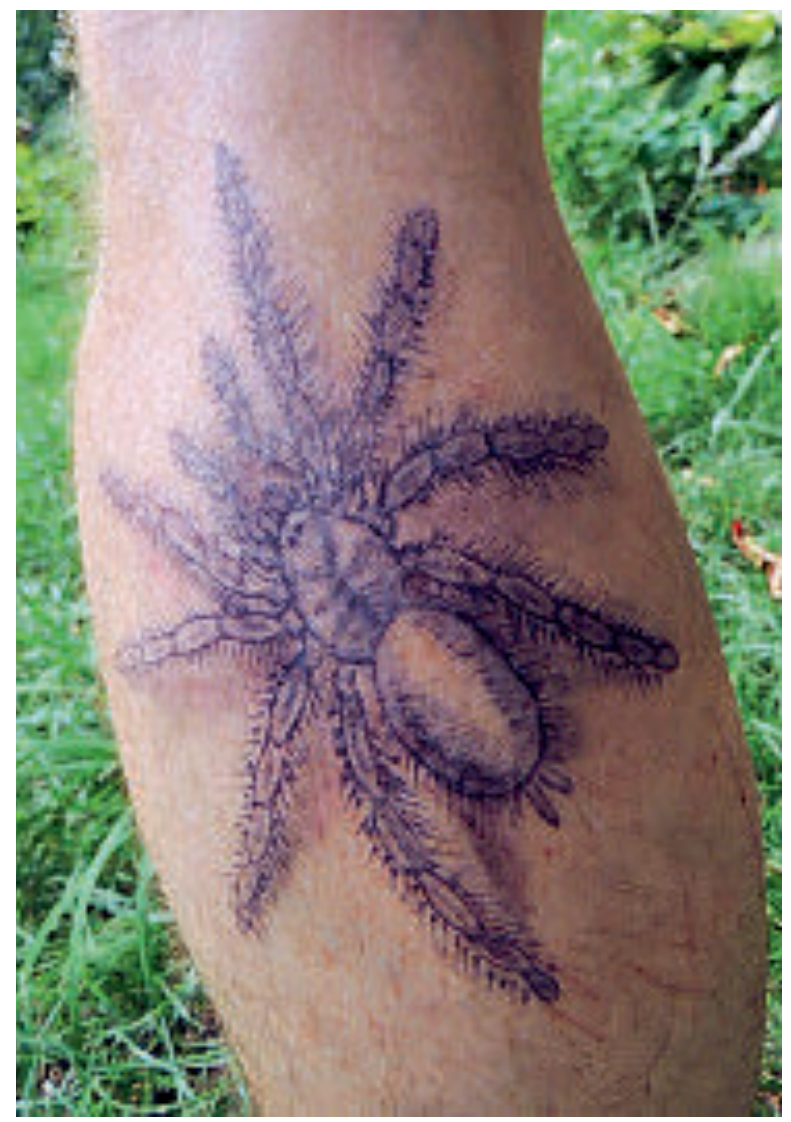

hochgeschlossene Kleidung und makellose Haut vorsieht, war das Schmücken des halbnackten Körpers mit Bemalung oder gar Tätowierung immer ein Zeichen des „Primitiven“, „Wilden“ - und übte genau dadurch auch immer einen prickelnden Reiz des geheimnisvoll Exotischen aus. Tatsächlich haben zu allen Zeiten und auf allen Kontinenten die Menschen ihre Haut mit Bemalung und Tätowierung geschmückt. Und auch wenn so etwas unzweifelhaft oft sehr cool und faszinierend aussieht, war der Zweck meist nicht einfach „Deko“, sondern vermittelte soziale Botschaften. Schon CaEsar berichtet in De Bello Gallico, wie die angreifenden Briten mit ihrer furchterregenden blauen Bemalung die Römer erschreckten. In anderen Fällen gibt der Körperschmuck Auskunft über Ehestand, Leistungen für das Gemeinwohl, Charaktereigenschaften oder errungene Siege. Und schließlich gehört es in vielen Kulturen zur Vorbereitung auf Festivitäten, einander aufwendig zu schmücken. Am bekanntesten ist sicher die Henna-Zeremonie bei orientalischen Hochzeiten, die von der Braut und ihren Freun- 


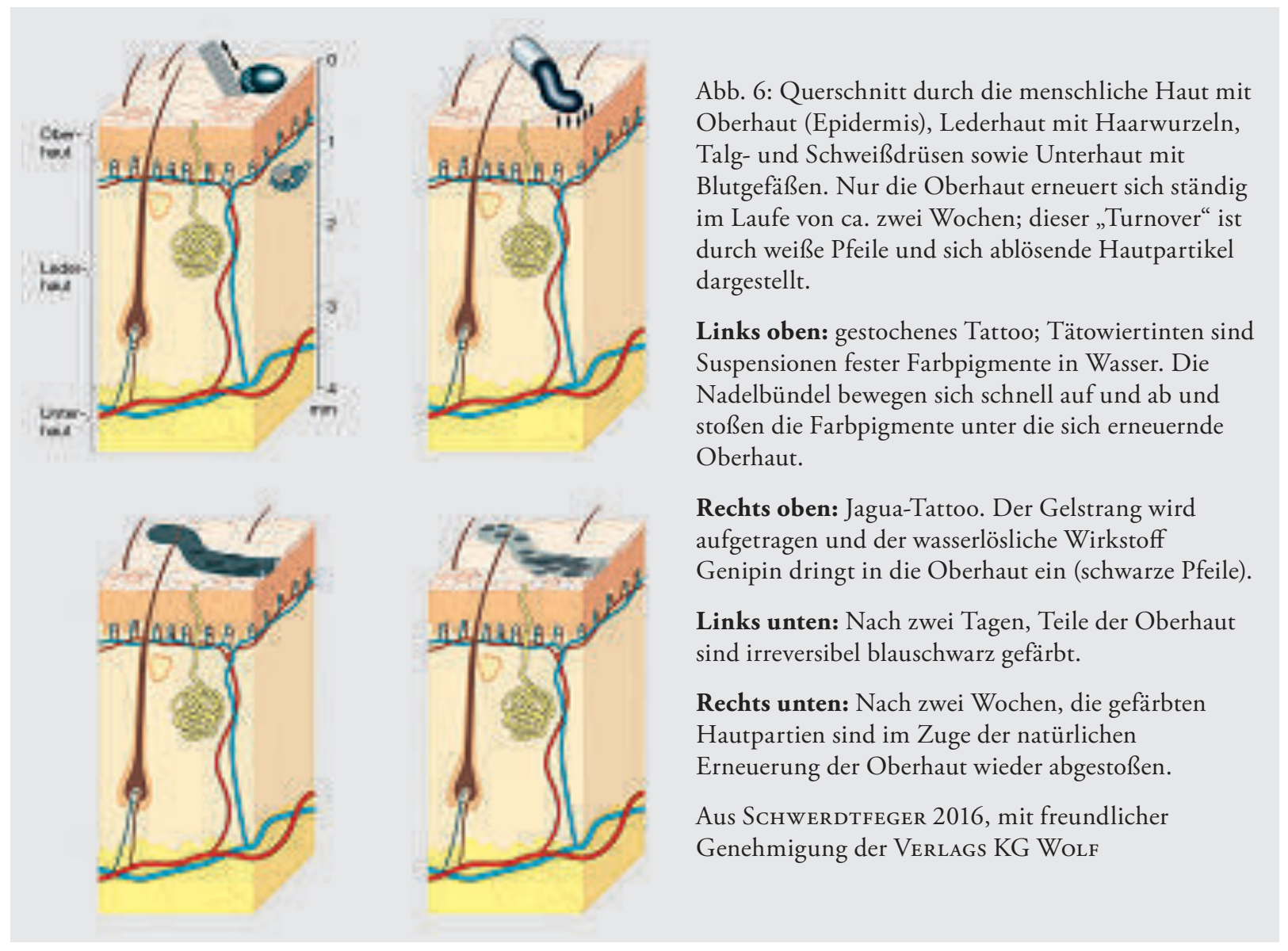

dinnen „strictly for women“ manchmal tagelang zelebriert und genossen wird.

Verhaltensforscher bezeichnen dieses entspannte Beisammensein und „Einander Pflegen“ im Tierreich als „Soziale Fellpflege“. Uns Zivilisierten ist es weitestgehend abhanden gekommen, sodass wir es oft nur noch in professionalisierter Form zulassen können und Geld für Friseur und Barbier, Maniküre, Massage oder Personal Trainer ausgeben.

Auf das tropische Amerika bezogen, bemerkt bereits Alexander von Humboldt in seinen Reiseerinnerungen, wie viel Zeit die Indigenen auf ihren Körperschmuck verwenden. Immer wieder berichtet er von rotem Onoto (Anatto, Achiote, Bixa orellana) und von der dauerhafteren, blauschwarzen Bemalung mit Caruto (Genipapó, Huito, Jagua, Genipa americana).

Wie ein Geschwisterpaar ziehen sich die rote Bixa und die schwarze Genipa durch den Hautschmuck des tropischen Amerika. Und tatsächlich kommen Manduru Warmi (Rote Frau) und Huito Warmi (Schwarze Frau) in einer Geschichte der Quichua als Geschwister daher, die sich der Welt in Form dieser beiden Pflanzenfarben schenken. (FABIUs et al. 2009).

Die mit Henna vergleichbare und sonst im gesamten Pflanzenreich wohl einzigartige Eigenschaft von Jagua: Der Wirkstoff Genipin dringt in die Oberhaut ein, geht mit den Eiweißen eine chemische Reaktion ein und bewirkt eine irreversible blauschwarze Färbung der Oberhaut, die nach einem Entwicklungsprozess von ein bis zwei Tagen ihren Höhepunkt erreicht und sich ebenso wenig abwaschen lässt wie ein echtes Tattoo. Während aber beim echten Tattoo die Nadeln die Farbpigmente ca. $1 \mathrm{~mm}$ unter die Oberfläche bringen, betrifft die Genipa-Färbung nur die Oberhaut, die sich kontinuierlich erneuert. Man kann sich darauf verlassen: Nach Ablauf dieses so genannten Turnover der Epidermis, also nach ca. 14 Tagen, ist das Jagua-Tattoo herausgewachsen. Wenn es allerdings gut gelungen ist (und man 
gar beginnt, das „Lebensgefühl Tattoo“ zu genießen), kann man das verblassende Design mit Jagua nachzeichnen und so die Lebensdauer seines Hinguckers immer um 14 Tage verlängern, solange man will.

\section{Wie Jagua in die Zivilisation kam}

Wir erleben seit den Neunziger Jahren einen Hype um den schönen, attraktiven Körper und eine nie dagewesene Renaissance des Tattoos, und Jagua passt perfekt in den Zeitgeist - wie vor ihm schon das Henna. Und so waren es Henna-Unternehmer, die das Jagua in die Zivilisation brachten. Das Künstlerehepaar CARINe Fabius und Pascal Giacomini, das in Hollywood eine Galerie hat, handelte seit den Neunziger Jahren mit Henna und entwickelte benutzerfreundliche Henna-Kits für die private Henna-Zeremonie zu Hause. Aber Henna sieht eben immer aus wie Henna und wollte nie etwas anderes sein: Es ist immer rotbraun, und die Designs sind floral-feminine, orientalischgeheimnisvolle Arabesken - für "coole Jungs“ kommt es also nicht in Frage. Aber dennoch gab es immer auch eine Nachfrage nach schwarzen temporären Tattoos, die man für echte halten kann, und so wurde das so genannte Schwarze Henna entwickelt. Bei diesem beruht jedoch die schwarzfärbende Wirkung auf dem chemischen Stoff Paraphenylendiamin (PPD), der bei vielen Personen schwere Hautreizungen, Entzündungen und Allergien hervorruft.

Die Suche nach dem gesundheitlich unbedenklichen schwarzen temporären Tattoo führte Carine und Pascal ins peruanische Amazonasgebiet zum Volk der Matsés, und Pascal reiste dorthin und konnte Zeuge von Ernte, Verarbeitung und Anwendung des Jagua werden. Daraus entwickelte sich eine stabile Geschäftsbeziehung, und bis heute sammeln die Matsés die Früchte von wildwachsenden Geni$p a$-Bäumen und schicken den Saft nach Los Angeles, wo er von Carine und Pascal zur Produktform weiterverarbeitet und unter dem Label EarthHenna weltweit vertrieben wird. Auch das bisher einzige Buch zum Thema Jagua stammt von CARINe (Fabius et al. 2009). Es ist jedoch nicht verwunderlich, dass sich der „Ge- heimtipp“ um die magische Indianertinte mittlerweile auch bei den Henna-Studios herumgesprochen hat und man Jagua längst auch von deutschen Anbietern beziehen kann.

\section{Frühe Zeugnisse von Jagua}

Bei dem derzeitigen Kult um den schönen Körper und der Allgegenwart des Massenphänomens Tattoo ist es erstaunlich, dass sich die Magische Indianertinte Jagua (Mittermeier in Fabius 2009) so lange quasi in den unzugänglichen Tropenwäldern vor uns verstecken konnte. Das L. im vollständigen wissenschaftlichen Namen deutet darauf hin, dass bereits Carl von Linné die Pflanze kannte und ihr den wissenschaftlichen Namen gab. Aber entdeckt hat auch er sie keineswegs. Schon hundert Jahre vorher findet sie als Ianipaba Erwähnung in der Historia Naturalis Brasiliae (Piso 1648). Nach einer ausführlichen Beschreibung der Pflanze heißt es dort:

„Nullum alium usum hic succus habet, ad nequitias dumtaxat exogitatus. Tinctura enim.. circa nonum diem evanescit; nulla alia ratione ablui potest" (PIso 1648) (Einen anderen Nutzen hat dieser Saft nicht, er dient also eigentlich nur zu nichtsnutzigem Müßiggang. Die Färbung verschwindet nämlich nach etwa neun Tagen, aber auf andere Weise ist sie nicht abzubekommen)

Kaum zu glauben: Der „Nichtsnutzige Müßiggang" mit der Pflanze für temporäre Tattoos ist den Europäern eigentlich schon fast 400 Jahre bekannt!

\section{Was für ein Potential!}

Aber wie „nichtsnutzig“ ist es eigentlich, seinen Körper zu schmücken? Freilich mag sich mancher sachlich-korrekte Zeitgenosse fragen, ob es nicht für „Wilde“ wie für „Zivilisierte“ Vordringlicheres gibt, als Zeit für eitle Selbstgestaltung aufzuwenden. Wer jedoch so argumentiert, möge sich vor Augen führen, wie viel Zeit die elegante Dame täglich in den perfekten Sitz ihrer Frisur und in das Schminken investiert, der smarte Banker in die makellos glatte Rasur, der selbstbewusste Bartträger in das tägliche Trimmen seines Gesichtsschmucks. Attraktivi- 


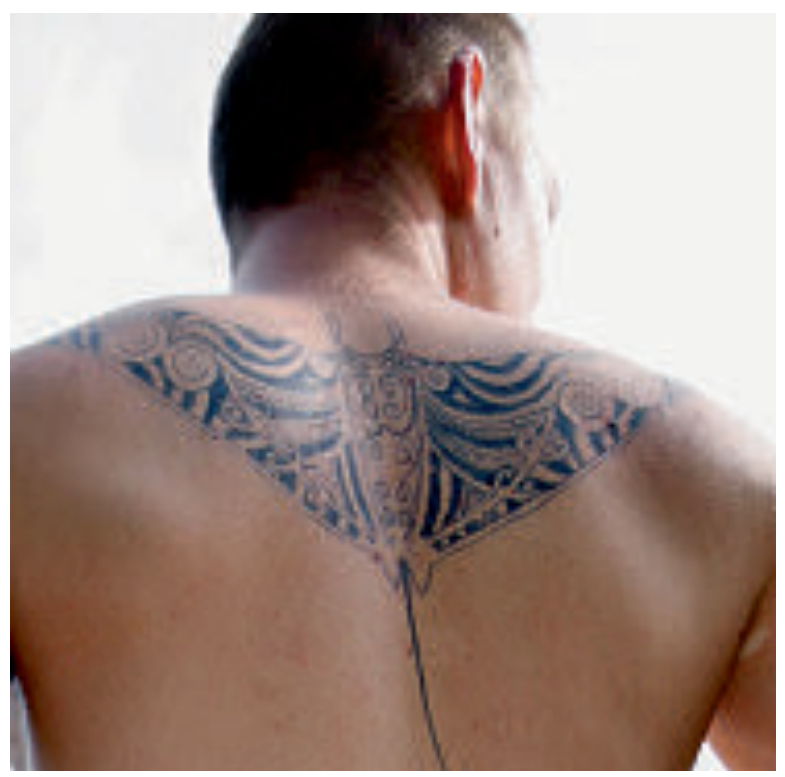

tät, Selbstgestaltung und Selbstdarstellung spielen in unserer Gesellschaft eine große Rolle (Lobstedt 2009), und die Welt der Cremes und Pflegeserien, der Sonnen- und Fitness-Studios, der Beautyfarms und der kosmetischen Chirurgie ist ein Milliardengeschäft. Auch die Tattooszene ist heute ein Wirtschaftsfaktor, und gute Künstler haben Wartezeiten von Monaten oder Jahren und Stundenlöhne, von denen viele andere Berufe nur träumen können. Die Vorbehalte der alten Generation, für die die Tätowierung oft etwas Rebellisches, Verruchtes, Halbkriminelles hatte, hat die Jugend überwunden. Nicht zuletzt gefördert durch die Medien, gehören für viele junge Menschen zum jugendlich-attraktiven Körper auch spektakuläre Tattoos, und so „holen“ sie sich oft früh und unüberlegt ihr erstes Tattoo, weil alle anderen im Freundeskreis auch schon eines haben. Diese Tattoo-Inflation, dieser Trend zum Tattoo als unverbindlichem Mainstream-Massenkonsumobjekt wird übrigens auch von vielen professionellen Tätowierern kritisch gesehen (Krause 2014), und die liebenswerte Vaterfigur der deutschen Tätowierer und Tätowierten, Herbert Hoffmann, sagte dazu schon vor fünfzehn Jahren „Ich wünsche den jungen Leuten, die den Wunsch nach einem Tattoo hegen, dass sie ihren wirklichen Neigungen und nicht unüberlegt Modetrends folgen" (Feige \& Krause 2000). Aber dieser Modetrend schafft freilich wieder einen Wirtschaftszweig: den der Laserkosmetik. Viele junge Leute geben nämlich ihre schönen und teuren Profi-Tattoos erstaunlich früh schon wieder zum Abschuss frei, wenn sie feststellen, dass sie sich vom Mainstream haben treiben lassen und es offenbar doch nicht „ihr“ Tattoo war.

Hier bietet Jagua das große Potenzial für Probetattoos. Für den einen ist es einfach ein Hingucker für Party, Strandurlaub, Rendezvous oder Bühnenauftritt. Für den anderen kann es ein Test sein: Wäre ein Tattoo etwas für mich? Welches Motiv, welcher Stil passen zu mir? Wo würde ich es tragen, bei welcher Gelegenheit soll es sichtbar bzw. muss es unsichtbar sein? Und wie fühlt es sich an, tätowiert zu sein? Das „Lebensgefühl Tattoo“ zum Ausprobieren!

Offenbar gehört es zur menschlichen Natur, seinen Körper zu schmücken. Doch ein Tattoo ist nicht für jeden etwas, und viele Nichttätowierte vermissen ihr Leben lang nichts. Für andere ist ein Tattoo etwas Großes, Feierliches, eine Zeremonie, ein Ritual, von dem Zauber und Faszination ausgehen. Jagua kann uns dabei helfen, unsere Position zu finden. Es ist viel mehr als nur eine alberne Bemalung. Irgendwie ist es die kleine Schwester des echten Tattoos und vermittelt auf seltsame Weise eine Anzahlung auf das „Lebensgefühl Tattoo“. Welch ein Potenzial! Detailliertere Infos in SchwerdTFEGER (2016).

\section{Literatur}

Fabius, C., Giacomini, P. \& Mittermeier, C. 2009: Jagua. A journey into body art from the amazon. Santo Domingo.

Feige, M. \& Krause, B. 2004: Tattoo- und PiercingLexikon. Kult und Kultur der Körperkunst. - Berlin. Krause, D. 2014: Goodbye Arschgeweih. Von der Kunst, beschissene Tätowierungen zu vermeiden. - München. LoвsтÄDт, T. 2011: Tätowierung, Narzissmus und Theatralität. Selbstwertgewinn durch die Gestaltung des Körpers. - Diss. Universität Duisburg-Essen. SchwerdtFeger, M. 2016: Jagua - Tattoos aus dem Regenwald. - Magdeburg.

Abb. 7: Jagua-Tattoo auf dem Rücken. 